\title{
Effects of Mixing Ratio of Binary Fine Particles on the Packing Density and Filtration Characteristics ${ }^{\dagger}$
}

\author{
Kuo-Jen Hwang * and Iou-Liang Lin \\ ${ }^{1}$ Department of Chemical and Materials Engineering, Tamkang University, Taiwan
}

\begin{abstract}
Binary fine particles were dispersed in glycerol aqueous solutions with different mixing ratios to study the effects of particle size distribution and fluid viscosity on the cake properties in dead-end filtration, such as average porosity, average specific filtration resistance and compressibility of cake. The average specific cake filtration resistance increases with increasing the fraction of small particles. However, the lowest cake porosity occurs under the volume fraction of large particles of 0.75 . Comparing different methods for porosity estimations, model estimation is more accurate for those particles near pure composition, while simulation method is more suitable for moderate composition of particle mixtures. Furthermore, the cake porosity increases but the average specific cake filtration resistance decreases with increasing fluid viscosity. An $18 \%$ porosity increase and a $30 \%$ filtration resistance decrease are obtained when fluid viscosity increases from 1 to $10 \times 10^{-3} \mathrm{~Pa} \cdot \mathrm{s}$. The cake properties, such as the particle packing structure in the cake and the resulting filtration resistance, are affected not only by the particle size distribution but also by the fluid viscosity. The particle size distribution plays a much more important role on the cake compressibility than the fluid viscosity does.
\end{abstract}

Keywords: particle size distribution, microfiltration, cake properties, fluid viscosity, packing porosity, filtration.

\section{Introduction}

Microfiltration has been widely used for separating fine particles from liquids in various industrial processes. In such a filtration, how to enhance filtration rate and separation efficiency are the most important issues on process engineers concern. In general, the cake properties, such as porosity and specific filtration resistance, are the most important parameters affecting filtration performance. The cake properties are determined mainly by many factors, for instance, the particle size distribution, fluid rheology and operating conditions. Therefore, understanding the effects of particle size distribution and fluid viscosity on the cake properties is an essential step in grasping a filtration problem.

Tiller F.M. et al. (1972) proposed power-type empirical equations to correlate cake porosity or specific filtration resistance with solid compressive pressure. The cake compressibility could be indicated by the exponent of those empirical equations. Lu W.M. and Hwang K.J.

Received 7 June 2015; Accepted 4 August 2015

J-STAGE Advance published online 12 September 2015

1 No.151, Yingzhuan Rd., Tamsui Dist., New Taipei City 25137, Taiwan

* Corresponding author: Kuo-Jen Hwang;

E-mail: kjhwang@mail.tku.edu.tw

TEL: +886-2-2621-5656 ext.2726 FAX: +886-2-2620-9887
(1993) employed a force balance model to discuss the critical friction angle for particle deposition on the cake surface in constant pressure filtration. The packing structure in cake could then be simulated using the friction angle under various conditions. When the critical friction angle was larger, the particles were more stable on the cake surface, the packing structure would be looser, and the cake porosity increased but the specific filtration resistance decreased. To discuss how the properties of cakes formed by dual-sized submicron particles affect the performance of cross-flow microfiltration, Hwang K.J. and Lin K.P. (2002) used packing theories of cavern and displacement effects to estimate the cake porosities with different particle compositions. Although the fluid flow effect in the tangential direction was ignored, the theoretical results agreed with the experimental data in the region near uniform sized particles.

The basic filtration equation was frequently used for estimating filtration flux based on the well-known Darcy's law, that is,

$$
q=\frac{\Delta P}{\mu\left(\mathrm{R}_{\mathrm{c}}+R_{\mathrm{m}}\right)}=\frac{\Delta P}{\mu\left(w_{\mathrm{c}} \cdot \alpha_{\mathrm{av}}+R_{\mathrm{m}}\right)}
$$

where $q$ is the filtration flux, $\Delta P$ is the filtration pressure, $\mu$ is the fluid viscosity, $w_{\mathrm{c}}$ is the cake mass, $\alpha_{\mathrm{av}}$ is the average specific cake filtration resistance, and $R_{\mathrm{c}}$ and $R_{\mathrm{m}}$ are the filtration resistances of filter cake and medium, 
respectively. According to this equation, the fluid viscosity is separated from cake filtration resistance. It implies that the cake properties are independent on the fluid flow behavior. The hypothesis is questionable because the particle packing is possible to be affected by the drag force exerted on the particles. This point will be discussed in this study.

Although the particle size distribution is believed an important factor affecting cake properties, few researchers devoted their efforts on the theoretical study of this issue. In this study, the effects of particle size distribution and fluid viscosity on the cake porosity and specific filtration resistance in dead-end microfiltration were studied. The cake properties under various conditions were estimated using theoretical models and simulation method, and were compared with experimental data.

\section{Estimation of cake properties}

\subsection{Models}

\subsubsection{Cake porosity}

Many models were derived for estimating the packing density of binary particles in the past 30 years (German, R.M., 1989), in which to consider the cavern and displacement effects are the simplest models for random packing. Cavern effect is used for the condition that few small particles are filled into the vacant space in a packed bed constructed by large particles. The packing porosity can then be estimated by a material balance (German R.M., 1989)

$$
\varepsilon_{\mathrm{av}}=\frac{\phi_{\mathrm{L}}-1+\varepsilon_{\mathrm{L}}}{\phi_{\mathrm{L}}}
$$

where $\phi$ is the volume fraction of particles, and the subscript L represents large particles. Another effect is considered that some small particles are replaced by few large particles in a packed bed constructed by most of small particles. The packing porosity in such a condition can also be estimated by a material balance (German R.M., 1989)

$$
\varepsilon_{\mathrm{av}}=1-\frac{1-\varepsilon_{\mathrm{S}}}{1-\varepsilon_{\mathrm{S}} \phi_{\mathrm{L}}}
$$

where the subscripts $S$ represent small particles. Because both cake porosity and particle volume fraction are smaller than 1, the cakes formed by dual-sized particles have smaller porosity than that by uniform-sized, and the difference becomes more obvious when the composition is far distant from uniform. The lowest porosity can then be given from the intersection point of the curves plotted using Eqs. (2) and (3), that is,

$$
\varepsilon_{\mathrm{av}}=\varepsilon_{\mathrm{s}} \cdot \varepsilon_{\mathrm{L}}
$$

\subsubsection{Specific cake filtration resistance}

According to Kozeny equation, the average specific filtration resistance of cake can be estimated using the following equation:

$$
\alpha_{\mathrm{av}}=\frac{k S_{\mathrm{o}}^{2} \cdot\left(1-\varepsilon_{\mathrm{av}}\right)}{\rho_{\mathrm{s}} \cdot \varepsilon_{\mathrm{av}}{ }^{3}}
$$

where $k$ is the Kozeny constant, and $S_{\mathrm{o}}$ is the specific surface area of particles. The theoretical Kozeny constant is 5.0 for a compact packed bed constructed by smooth spheres. For a random packing, the relationship between Kozeny constant and packing porosity can be derived theoretically using the free cell model (Happel J. and Brenner H., 1965) as:

$$
k=\frac{2 \varepsilon^{3}}{(1-\varepsilon) \cdot\left\{\ln \left[\frac{1}{(1-\varepsilon)}\right]-\frac{\left[1-(1-\varepsilon)^{2}\right]}{\left[1+(1-\varepsilon)^{2}\right]}\right\}}
$$

The mean particle diameter, $d_{\mathrm{p}, \mathrm{av}}$ for dual-sized particles can be calculated by the primary sizes and the volume fractions of particles, that is,

$$
\frac{1}{d_{\mathrm{p}, \mathrm{av}}}=\frac{\phi_{\mathrm{L}}}{d_{\mathrm{p}, \mathrm{L}}}+\frac{\phi_{\mathrm{S}}}{d_{\mathrm{p}, \mathrm{S}}}
$$

Because the specific surface area exactly equals $6 / d_{\mathrm{p}}$ for a spherical particle, the value of $S_{\mathrm{o}}$ for a particle mixture can be estimated using the mean particle diameter as following:

$$
S_{\mathrm{o}}=\frac{6}{d_{\mathrm{p}, \mathrm{av}}}
$$

Once the variables in Eq.(5), such as $k, S_{\mathrm{o}}$ and $\varepsilon_{\mathrm{av}}$, are known, the average specific filtration resistance of cake can be calculated.

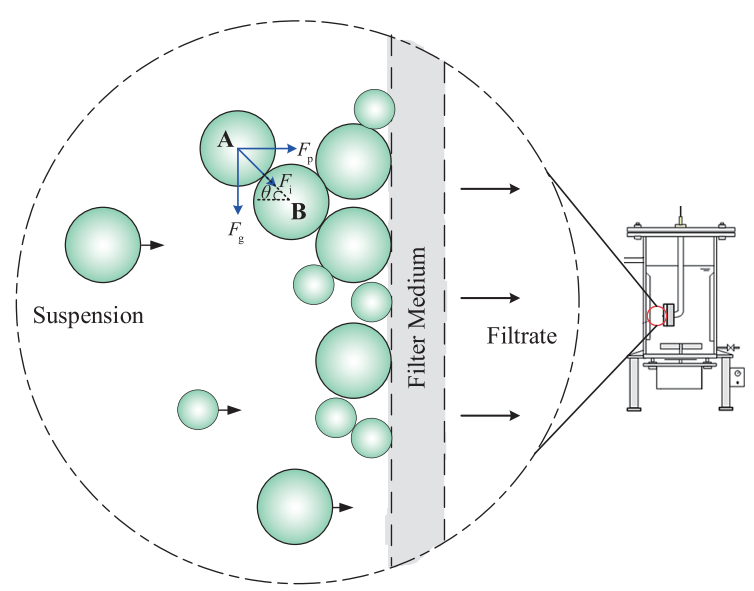

Fig. 1 Forces exerted on a depositing particle in microfiltration. 


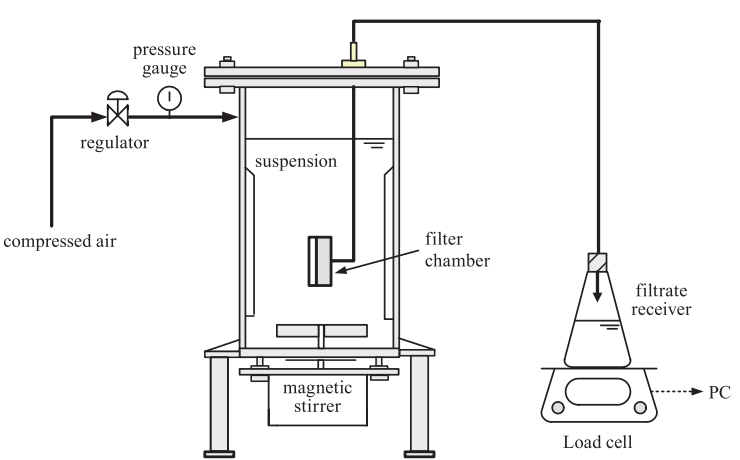

A numerical method for simulating the particle packing structure and cake porosity in dead-end filtration was proposed by Lu W.M. and Hwang K.J. (1993). It was modified to be used for the system of this study. Refer to Fig. 1, the external forces exerted on a depositing particle, Particle A, include the drag force due to permeate flow, $F_{\mathrm{p}}$, the net inter-particle force, $F_{\mathrm{i}}$, and the net gravitational force, $F_{\mathrm{g}}$. The drag force can be estimated using the modified Stokes law (Lu W.M. and Hwang K.J., 1993). The inter-particle force can be estimated using the DLVO theory based on a pair-wise assumption. According to this theory, the net inter-particle force is a summation of van der Waals force and electrostatic force (Hwang K.J. et al., 1997).

The Newton's second law of motion is integrated to estimate the velocity and the displacement of particle migration during filtration. Particle migrations are simulated until they can deposit stably. The details can be referred to Lu W.M. and Hwang K.J. (1993, 1995).

\section{Materials and methods}

Two uniform-sized polymethyl methacrylate (PMMA) spherical particles with a density of $1210 \mathrm{~kg} / \mathrm{m}^{3}$ were used in experiments. The particles were purchased from Soken Co., Japan. Their density was $1210 \mathrm{~kg} / \mathrm{m}^{3}$, and their mean diameters were $0.45 \mu \mathrm{m}$ (Ca.\#: MP-1000) and $5.0 \mu \mathrm{m}$ (Ca.\#: MX-500), respectively. Their particle size distributions were measured using a HORIBA LA-300 laser particle size analyzer and are shown in Fig. 2. Propane-1,2,3triol (glycerol) with a density of $1255 \mathrm{~kg} / \mathrm{m}^{3}$ and purity of $99.5 \%$ in volume was purchased from Fisher Scientific Co. in USA (Ca.\#: G33-4). Different amounts of glycerol were added into de-ionized water to prepare aqueous

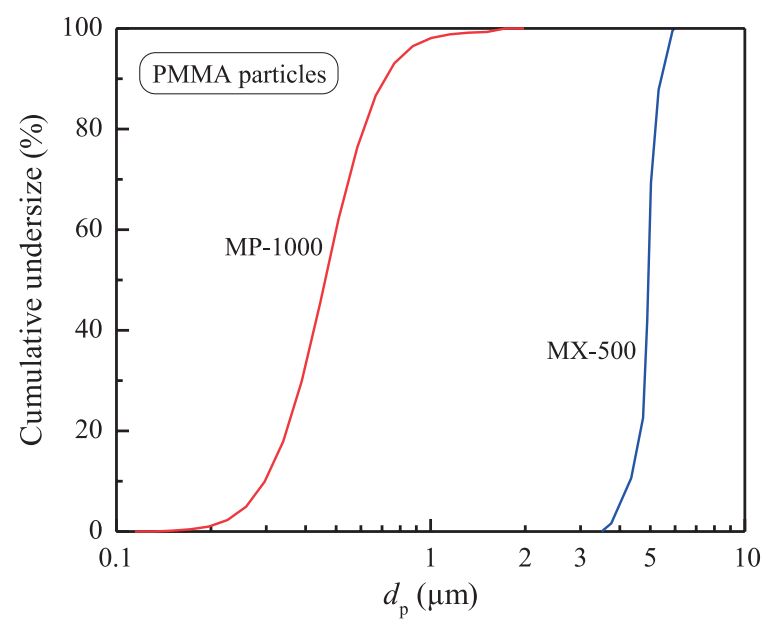

Fig. 2 Size distributions of particles used in this study.

solutions with different viscosities. The fluid viscosity was measured using a viscometer (Brookfield LVDV-II CP, USA). The particle suspensions with a concentration of $3 \mathrm{~kg} / \mathrm{m}^{3}$ were prepared by dispersing particles into those aqueous solutions under different particle mixing ratios. The particle size distributions were then adjusted by different mixing ratios of the dual-sized particulate samples. The $\mathrm{pH}$ and temperature of the suspension were kept at 7.0 and $20{ }^{\circ} \mathrm{C}$, respectively. The zeta potential of particles under such a condition was measured as $-25 \mathrm{mV}$. A membrane manufactured by ADVANTEC Co. in Japan (Ca.\#: A010A142C) was used in filtration as filter medium. The membrane was made of mixed cellulose ester, its mean pore size was $0.1 \mu \mathrm{m}$, and its filtration resistance was $2.26 \times 10^{11} \mathrm{~m}^{-1}$ under a filtration pressure of $2 \times 10^{4} \mathrm{~N} / \mathrm{m}^{2}$.

Dead-end microfiltration experiments were performed using a bomb filter shown in Fig. 3. The filtration area in the filter chamber was $8.04 \times 10^{-4} \mathrm{~m}^{2}$. Suspension was prepared in the filter and prevented particle settling using a magnetic stirrer. The filter chamber was installed to orient to the filter wall, as shown in Fig. 3, to prevent the obstruction or destruction of cake formation by stirring. The filtration pressure was supplied by a compressed air, adjusted using a regulator, and indicated by a pressure gauge. The filtrate was received into a flask and weighed using a load cell. The data of filtrate weight was transferred to volume and recorded on a personal computer during the whole course of filtration. An orifice plate was installed in the filter chamber to indicate the cake growth. When the growing cake surface reached the orifice plate, the filtration area decreased suddenly, and the tangent slope of the filtration curve of $\mathrm{d} t / \mathrm{d} v$ vs. $v$ increased drastically. Therefore, the average cake porosity could be calculated using the received filtrate volume based on a mass balance, and the average specific cake filtration resistance was determined by the tangent slope of the filtration curve before the sudden change. 


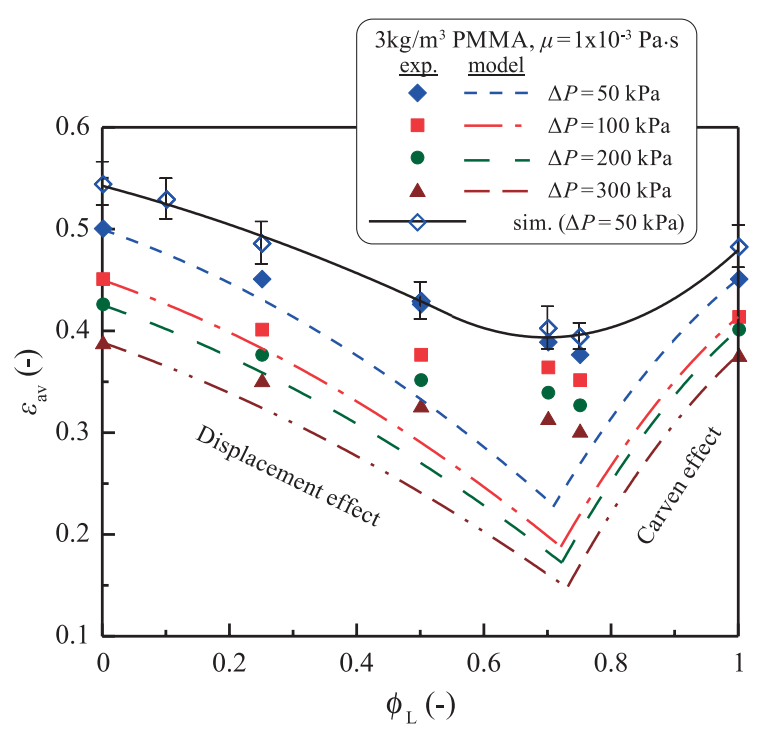

\section{Results and discussion}

Fig. 4 shows the filtration curves of $\mathrm{d} t / \mathrm{d} v$ versus $v$ under various filtration pressures for uniform PMMA particle with a mean size of $0.4 \mu \mathrm{m}$. The dispersion medium was de-ionized water with a viscosity of $1 \times 10^{-3} \mathrm{~Pa} \cdot \mathrm{s}$. The filtration curves have the same trend; each of them can be divided into two parts. The first part is a straight line. This implies that the average specific cake filtration resistance is constant during filtration. When the filter chamber is filled with cake, the received filtrate volume reaches $v_{\mathrm{f}}$, and the tangent slope of the filtration curve suddenly increases due to the decrease in filtration area. The average specific cake filtration resistance is then calculated by the slope of the straight line before the transition point, as those shown in the figure. The average cake porosity can be obtained using the mass balance at the transition point because the cake volume is known. Comparing the values of $v_{\mathrm{f}}$ under various pressures, a smaller $v_{\mathrm{f}}$ value under lower pressure indicates a higher cake porosity in such condition. Furthermore, because the value of $\mathrm{d} t / \mathrm{d} v$ is the reciprocal of filtration flux, the curves shown in Fig. 4 also reveal that a lower filtration pressure results in a lower filtration flux.

Fig. 5 shows the comparisons of cake porosity among the experimental data, simulated results and model calculated results under various large particle fractions and various filtration pressures. The dispersed medium was de-ionized water with a viscosity of $1 \times 10^{-3} \mathrm{~Pa} \cdot \mathrm{s}$. The values of cake porosity of dual-sized particles are lower than those of mono-sized under a given filtration pressure, and the lowest cake porosity occurs around the large particle fraction $\phi_{\mathrm{L}}=0.75$. When a few large particles exist in a small particle packing, some original packing positions of small particles are replaced by large particles, and the cake porosity due to this "displacement effect" can be estimated using Eq. (3). The cake porosity decreases with

Fig. 5 Comparisons of cake porosity among model estimations, simulated results, and experimental data for various mixing fractions of large particles.

increasing the volume fraction of larger particles. On the other hand, when a small portion of small particles packs into a cake constructed by large particles, small particles may pack into the void space among large particles, and the cake porosity due to the "carven effect" can be estimated using Eq. (2). This effect causes the cake porosity decreases with increasing the fraction of small particles. The curves calculated using Eqs. (2) and (3) show that the lowest cake porosities occurring at $\varepsilon_{\mathrm{L}} \varepsilon_{\mathrm{S}}$ where is the intersection point of those two curves, i.e, Eq. (4). These results agree qualitatively with the experimental data. However, the estimated results are always lower than experimental data, and obvious deviations between them can be found under moderate fractions of large (or small) particles. The maximum deviation is as high as $30 \%$ occurring at the lowest cake porosity. This demonstrates that the models of cavern and displacement effects can be used properly only when a few different-sized particles are added into a cake formed by uniform-sized particles. Those models were originally used for estimating the random packing of particles under gravity. All particles have opportunities to migrate to reach stable positions. This is similar to the conditions under high filtration rates. However, when filtration rate is not high enough, the depositing particles may have no enough drag force to push them to form a compact cake. Thus, the packing porosity increases with decreasing filtration rate. This is therefore another reason why the deviation between the model estimations and experimental data becomes larger for a more compact cake. Furthermore, comparing the cake porosities under various filtration pressures at a fixed volume fraction of large particles, an increase in filtration pressure leads to lower cake porosity. It is attributed to more 


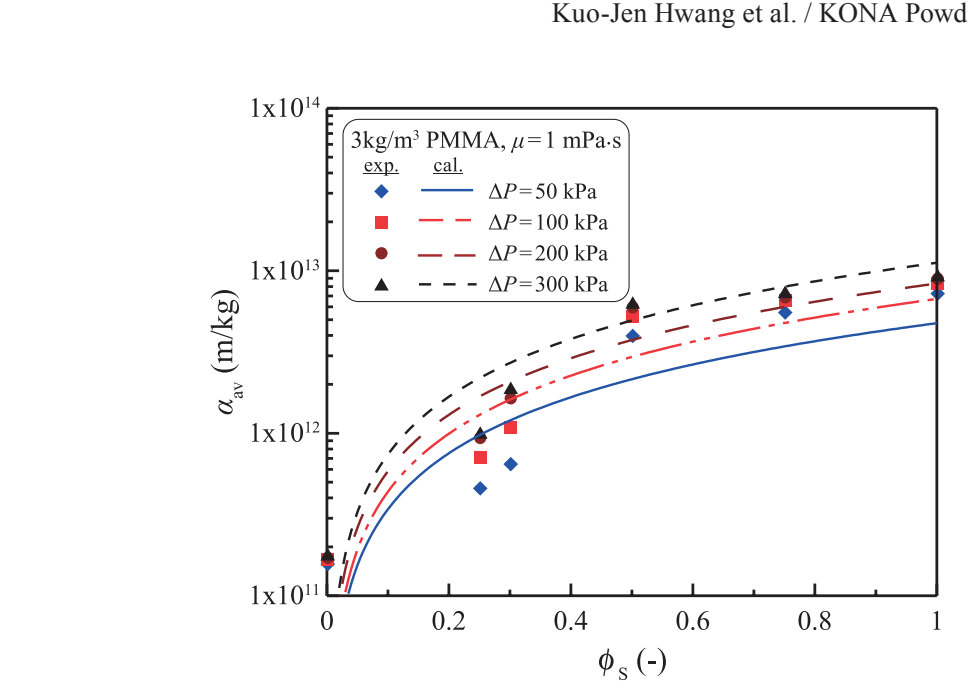

Fig. 6 Comparisons of average specific cake filtration resistance between calculated results and experimental data for various mixing fractions of small particles.

severe cake compression under a higher pressure. Another method for cake porosity estimation is computer simulation. Although the simulation results are scattered, the trend of the simulated curve is much closer to that of experimental data. Comparing those estimations obtained by different methods, model estimation is more accurate for those particles near pure composition, while simulation method is more suitable for moderate composition of particle mixtures, especially for the compositions to result in the lowest cake porosity.

Fig. 6 shows the effects of small particle fraction, $\phi_{\mathrm{S}}$, and filtration pressure, $\Delta P$, on the average specific cake filtration resistance, $\alpha_{\mathrm{av}}$. The theoretical curves were calculated by substituting the calculated average cake porosity into Eq. (5), in which the values of Kozeny constant and specific surface area of particles were estimated using Eqs. (6)-(8), respectively. Comparing those factors affecting specific filtration resistance, specific surface area and particle size play the most important roles. The mean particle size decreases from 5 to $1 \mu \mathrm{m}$ as $\phi_{\mathrm{S}}$ value increases from 0 to 0.3 . However, there has only a $50 \%$ decrease in the mean particle size when $\phi_{\mathrm{S}}$ increases from 0.3 to 1.0 . Therefore, the value of $\alpha_{\mathrm{av}}$ increases very rapidly with increasing $\phi_{\mathrm{S}}$ when $\phi_{\mathrm{S}}$ value is smaller than 0.3 . The slight increase in cake porosity and decrease in mean particle size with $\phi_{\mathrm{S}}$ cause the $\alpha_{\mathrm{av}}$ values to become nearly invariant when $\phi_{\mathrm{S}}>0.5$. The calculated results agree with experimental data except for the overestimations occurring around $\phi_{\mathrm{S}}=0.3$. This is attributed to the marked underestimations of cake porosity at that particle mixing fraction, as shown in Fig. 5.

The experimental data of average specific cake filtration resistance are plotted against filtration pressure for different large particle fractions in logarithm scales in Fig. 7. The data can be regressed to a straight line for a given particle mixing fraction. This reveals that the rela-

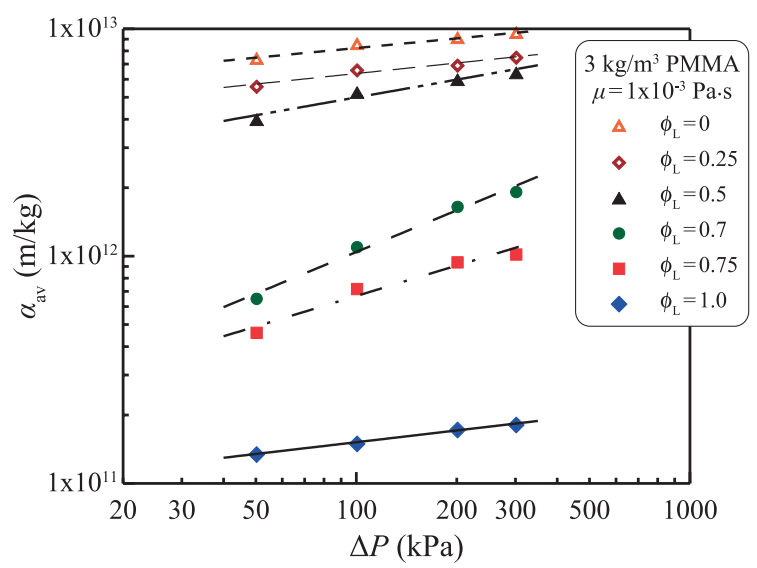

Fig. 7 Plots of $\alpha_{\mathrm{av}}$ versus $\Delta P$ under various mixing fractions of large particles.

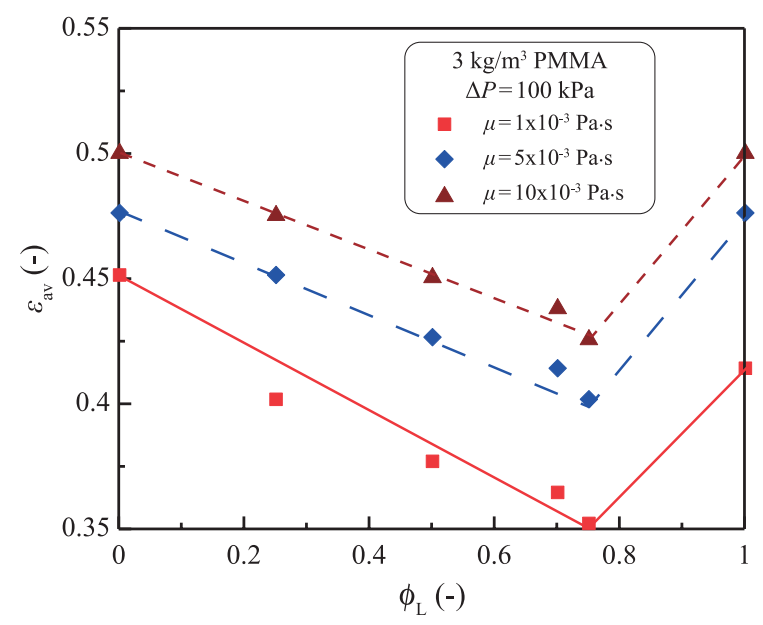

Fig. 8 Effects of fluid viscosity and particle mixing fraction on the average cake porosity.

tionship between $\alpha_{\mathrm{av}}$ and $\Delta P$ follows a power function proposed by Tiller et al. (1980). The $\alpha_{\mathrm{av}}$ value increases with increasing pressure and decreasing the fraction of large particles. The $\alpha_{\mathrm{av}}$ value of pure small particles is $c a$ two order-of-magnitudes higher than that of pure large particles under a given pressure. This is because the $\alpha_{\mathrm{av}}$ value is proportional to the inverse square of particle size. According to the Tiller's empirical equation, the slope of a curve represents the cake compressibility. Comparing the slopes of the curves shown in the figure, the cake compressibility is very low (around 0.07) for uniform-sized particles and shows the highest value around 0.55 occurring at the fraction of large particles of 0.7 . This result indicates that the cake compressibility is sensitive to particle size distribution and will be discussed in more detail later.

The viscosity of dispersed fluid was adjusted by adding different amounts of glycerol into de-ionized water. The aqueous solutions were measured as Newtonian fluids, and the values of viscosity were 10 and $5 \times 10^{-3} \mathrm{~Pa} \cdot \mathrm{s}$ for 


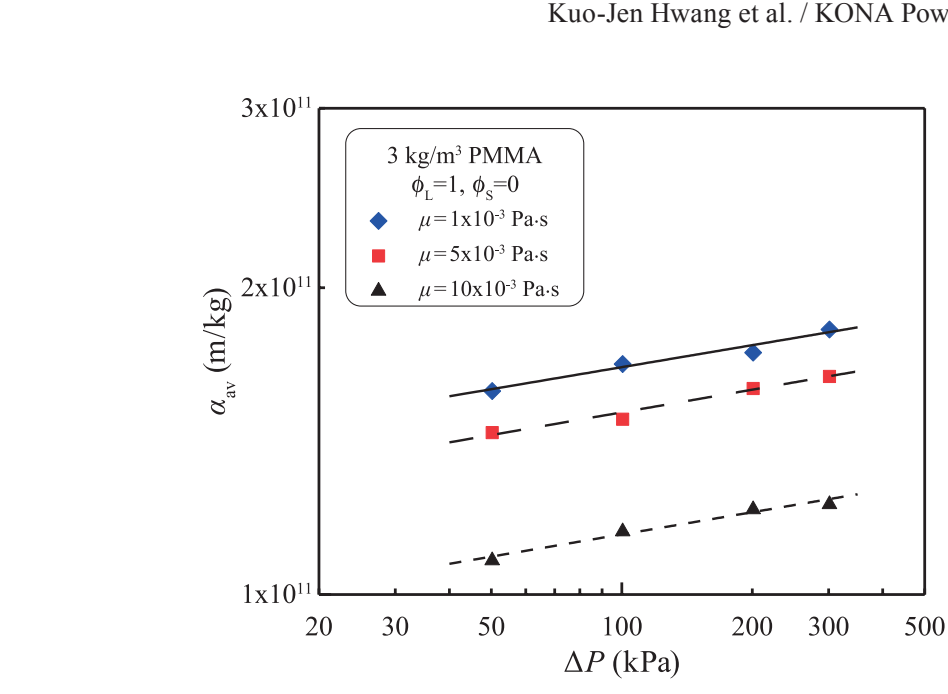

Fig. 9 Plots of $\alpha_{\mathrm{av}}$ versus $\Delta P$ under various fluid viscosities.

53 and 45 vol\% glycerol solutions, respectively. The same viscosity values measured before and after filtration experiments demonstrate that no glycerol molecule was retained on or adsorbed onto the particles, cake or filter media during filtration. Fig. 8 shows the effects of fluid viscosity and volume fraction of large particles on the average cake porosity. The curve trend for each fluid viscosity is similar to that in Fig. 5. The values of cake porosity are higher for uniform-sized particles, and a minimum cake porosity occurs at $\phi_{\mathrm{L}}=0.75$. An increase in fluid viscosity leads to higher cake porosity. An $18 \%$ porosity increase is obtained as fluid viscosity increases from 1 to $10 \times 10^{-3} \mathrm{~Pa} \cdot \mathrm{s}$. This is because a higher fluid viscosity causes a larger drag force exerted on the depositing particles as well as larger surface friction between particles. As a result, the particle packing structure in a filter cake is looser.

Fig. 9 shows the effects of fluid viscosity and filtration pressure on the average specific cake filtration resistance for pure large particles. The relationship between $\alpha_{\mathrm{av}}$ and $\Delta P$ can be regressed to straight lines, i.e., power-type Tiller's empirical equations, the same as those mentioned in Fig. 7. Because the cake porosity is lower (a more compact cake) for a dispersed fluid with lower viscosity, the $\alpha_{\mathrm{av}}$ value is therefore higher. A $30 \%$ filtration resistance decrease is obtained when fluid viscosity increases from 1 to $10 \times 10^{-3} \mathrm{~Pa} \cdot \mathrm{s}$. This magnitude variation in $\alpha_{\mathrm{av}}$ can be reasonably explained by the particle packing structure for a given particle size distribution. Thus, the $\alpha_{\mathrm{av}}$ value is not a sole function of particles themselves; but it is also affected by the physical properties of dispersed fluid, e.g., fluid viscosity. This result is different from those previous inferences in which the resistance caused by dispersed medium, fluid viscosity, is separated from cake resistance term, e.g., $\alpha_{\mathrm{av}}$. In conclusion, the influence of fluid properties on the overall filtration resistance cannot be separated to a single term of fluid viscosity. The cake properties, at

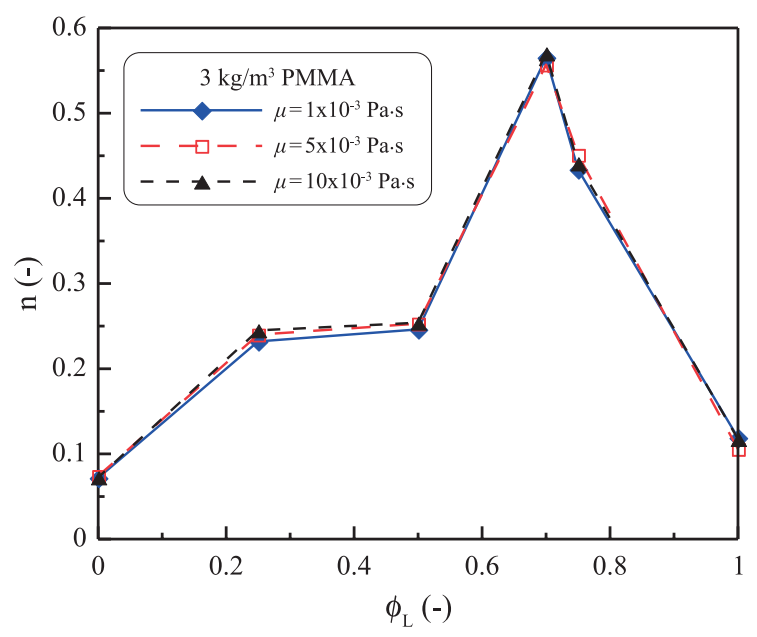

Fig. 10 Effects of particle mixing fraction and fluid viscosity on the cake compressibility.

least the particle packing structure in the cake and the resulting filtration resistance, are also affected by fluid viscosity. However, the similar slopes of the curves shown in the figure indicate that the cake compressibility is similar and independent of fluid viscosity.

The cake compressibility can be obtained from the line slope plotted by $\alpha_{\mathrm{av}}$ vs. $\Delta P$ in logarithm scales, as those shown in Figs. 7 and 9. The effects of particle mixing fraction and fluid viscosity on the cake compressibility are shown in Fig. 10. The particle size (distribution) plays a much more important role on the cake compressibility than the fluid viscosity does. Although the average specific cake filtration resistance was affected obviously by the fluid viscosity, the cake compressibility was nearly constant for a given particle mixing fraction. For those uniform-sized conditions, the values of cake compressibility are as low as 0.1 ; and the cakes are only slightly compressible. The cake compressibility becomes higher in the conditions of particles with size distributions. This is because particles have more opportunity to pack into the voids to form more compact structure during filtration. The highest cake compressibility is $c a 0.56$ occurring at the mixing fraction of large particles of 0.70 . This particle mixing fraction is a little different from that resulting in the lowest cake porosity $\left(\phi_{\mathrm{L}}=0.75\right.$, as those shown in Fig. 8). It is because the factors affecting the specific cake filtration resistance is not only the cake porosity but also the particle size distribution.

\section{Conclusions}

The effects of particle size distribution, fluid viscosity and filtration pressure on the average cake porosity, average specific cake filtration resistance, and cake compressibility in dead-end constant pressure microfiltration were 
stardied ${ }^{2}$ An increase in filtration pressure led to lower ayefage cake porosity and higher specific cake filtration resistance. The effect of particle mixing fraction on the cake porosity was explained using packing models, such as cavern and displacement effects. The lowest cake porosity occurred under the volume fraction of large particles of 0.75 . Model estimation for cake porosity was more accurate for those particles near pure composition, while simulation method was more suitable for moderate composition of particle mixtures, especially for the compositions resulting in the lowest cake porosity. The average specific cake filtration resistance increased with increasing the fraction of small particles, especially for low fraction of small particles. Furthermore, the cake properties were affected by fluid viscosity. The cake porosity increased but the average specific cake filtration resistance decreased with increasing fluid viscosity. An $18 \%$ porosity increase and a $30 \%$ filtration resistance decrease were obtained when fluid viscosity increases from 1 to $10 \times 10^{-3} \mathrm{~Pa} \cdot \mathrm{s}$. This was attributed to the larger surface friction causing by higher viscous flow. The cake properties, such as the particle packing structure in the cake and the resulting filtration resistance, were indeed affected not only by particle size distribution but also by fluid viscosity. The particle size distribution played a much more important role on the cake compressibility than the fluid viscosity does, and the highest cake compressibility occurred approximately at the particle mixing fraction resulting in the lowest cake porosity.

\section{Acknowledgements}

The authors wish to express their sincere gratitude to the Ministry of Science and Technology of the Republic of China for its financial support (Grant number: MOST 103-2221-E-032-057-MY3).

\section{Nomenclature}
$d_{\mathrm{p}} \quad$ particle diameter $(\mu \mathrm{m})$
$F_{\text {g }}$ net gravitational force $(\mathrm{N})$
$F_{\text {i }} \quad$ net inter-particle force $(\mathrm{N})$
$F_{\mathrm{p}} \quad$ drag force due to permeate flow (N)

$k \quad$ Kozeny constant $(-)$

$\Delta P$ filtration pressure $(\mathrm{Pa})$

$q \quad$ filtration flux $\left(\mathrm{m}^{3} / \mathrm{m}^{2} \mathrm{~s}\right)$

$R_{\mathrm{c}} \quad$ filtration resistance of cake $\left(\mathrm{m}^{-1}\right)$

$R_{\mathrm{m}} \quad$ filtration resistance of medium $\left(\mathrm{m}^{-1}\right)$

$S_{\mathrm{o}} \quad$ specific surface area of particles $\left(\mathrm{m}^{2} / \mathrm{m}^{3}\right)$

$t \quad$ filtration time (s)

$v$ filtrate volume per unit filtration area $\left(\mathrm{m}^{3} / \mathrm{m}^{2}\right)$

$v_{\mathrm{f}} \quad$ filtrate volume per unit filtration area at which the growing cake surface reached the orifice plate $\left(\mathrm{m}^{3} / \mathrm{m}^{2}\right)$

$w_{\mathrm{c}} \quad$ cake mass $\left(\mathrm{kg} / \mathrm{m}^{2}\right)$

$\alpha \quad$ specific filtration resistance of cake $(\mathrm{m} / \mathrm{kg})$

$\varepsilon \quad$ cake porosity (-)

$\phi \quad$ volume fraction of particles (-)

$\mu \quad$ fluid viscosity $(\mathrm{Pa} \cdot \mathrm{s})$

\section{Subscripts}

av average cake property

L large particle

S small particle

\section{References}

German R.M., Particle Packing Characteristics, Chap. 6, Metal Powder Industries Federation, Princeton, New Jersey, USA, 1989.

Happel J., Brenner H., Low Reynolds Number Hydrodynamics, Englewood Cliffs, NJ, Prentice Hall, 1965.

Hwang K.J., Yu M.C., Lu W.M., Migration and deposition of submicron particles in crossflow microfiltration, Separation Science and Technology, 32 (1997) 2723-2747.

Hwang, K.J., Lin, K.P., Cross-Flow Microfiltration of dual-sized submicron particles, Separation Science and Technology, 37 (2002) 2231-2249.

Lu W.M., Hwang K.J., Mechanism of cake formation in constant pressure filtrations, Separation Technology, 3 (1993) 122-132.

Lu W.M., Hwang K.J., Cake formation in 2-D cross-flow filtration, A.I.Ch.E. Journal, 41 (1995) 1443-1455.

Tiller F.M., Haynes S., Lu W.M., The role of porosity in filtration VII Effect of side-wall friction in compression-permeability cells, A.I.Ch.E. Journal, 18 (1972) 13-20. 


\section{Author's short biography}

\section{Kuo-Jen Hwang}

Dr. Kuo-Jen Hwang is now a Professor at Tamkang University, Taiwan. After getting his PhD degree in National Taiwan University in 1992, he moved to the Department of Chemical Engineering at Tamkang University, as an Associate Professor. He became a full professor at Tamkang University in 1997. He was appointed as the Chairman of the Department of Chemical Engineering, Tamkang University from 1997 to 2001.

His research interests focus on solid-liquid separation and powder technology, especially for microfiltration of fine and soft particles, and hydrocyclone for separation and classification of fine particles. He has published over 100 papers in peer reviewed journals and 150 conference papers. He is also the Chairmen of the Taiwan Filtration and Separations Society and the International Delegates on Filtration since 2012.

\section{Iou-Liang Lin}

Mr. Iou-Liang Lin received his MS degree at the Department of Chemical and Materials Engineering, Tamkang University in 2009. He is a System Engineer in INNOLUX Corporation, Taiwan now. 